\title{
Racial Treatment Trends in Localized/Regional Prostate Carcinoma: 1992-1999
}

\author{
Willie Underwood III, M.D., M.S., M.P..H. ${ }^{1,2}$ \\ James Jackson, Ph.D. ${ }^{3,4}$ \\ John T. Wei, M.D., M.s. ${ }^{1}$ \\ Rodney Dunn, m.s. ${ }^{1}$ \\ Edmond Baker, M.D. ${ }^{5}$ \\ Sonya DeMonner, M.P.H. ${ }^{6}$ \\ David P. Wood, M.D. ${ }^{1}$
}

${ }^{1}$ Division of Clinical Research and Quality Assurance, Department of Urology, University of Michigan, Ann Arbor, Michigan.

${ }^{2}$ Veterans Administration Center for Practice Management and Outcomes Research, Ann Arbor, Michigan.

${ }^{3}$ Research Center for Group Dynamics, Institute for Social Research, University of Michigan, Ann Arbor, Michigan.

${ }^{4}$ Center for African American and African Studies, University of Michigan, Ann Arbor, Michigan.

${ }^{5}$ Department of Surgery, University of Michigan, Ann Arbor, Michigan.

${ }^{6}$ Robert Wood Johnson Clinical Scholars Program, University of Michigan, Ann Arbor, Michigan.

Supported by United States Public Health Service Grant P50CA69568 (National Cancer Institute).

Address for reprints: Willie Underwood III, M.D., M.S., M.P.H., Department of Urology, University of Michigan, 1500 East Medical Center Drive, Ann Arbor, Ml 48109-0330; Fax: (734) 647-3301; Email: wunderwo@med.umich.edu

Received June 3, 2004; revision received September 7, 2004; accepted 0ctober 6, 2004.

\begin{abstract}
BACKGROUND. African-American men have a greater incidence of and mortality from prostate carcinoma compared with white men, and they are less likely to receive definitive therapy (radical prostatectomy or external beam radiation therapy). During the 1990s, the use of brachytherapy increased; however, its influence on racial and ethnic prostate carcinoma treatment trends remains unclear. The objective of this study was to describe treatment trends over the period 1992-1999 for localized/regional prostate carcinoma among white, Hispanic, and AfricanAmerican men.
\end{abstract}

METHODS. Using the Surveillance, Epidemiology, and End Results (SEER) registry data from 1992 through 1999, logistic regression models were used to determine whether the odds of receiving a specific treatment modality differed by racial and ethnic group and whether the differences changed over time when the models were adjusted for age, marital status, tumor grade, and SEER site (geography).

RESULTS. The authors identified 142,340 men, including white men (81.6\%), Hispanic men (6.4\%), and African-American men (12.0\%). Racial and ethnic differences in the rates of use of androgen-deprivation therapy/expectant management were noted; however, these differences appeared to lessen over time $(P<0.001)$. The rate of utilization of radical prostatectomy increased for Hispanic men, remained flat for African-American men, and decreased for white men. The utilization of brachytherapy and combination therapy increased for all three groups; however, the greatest increase in utilization was among white men.

CONCLUSIONS. Further research will be required to determine the patient-level and provider-level variables that influence racial and ethnic treatment differences in localized/regional prostate cancer. Cancer 2005;103:538-45.

(ㄷ) 2004 American Cancer Society.

KEYWORDS: African American, Hispanic, white, brachytherapy, radical prostatectomy, external beam radiation therapy, prostate carcinoma, prostate carcinoma treatment, combination therapy.

D rostate carcinoma is the most prevalent noncutaneous malignancy in American men. It is projected that there were 220,900 incident diagnoses of prostate carcinoma and 28,900 deaths in the United States in 2003, accounting for $30 \%$ of all newly diagnosed malignancies. ${ }^{1}$ Compared with white men, African-American men have a greater incidence and mortality from prostate carcinoma, ${ }^{1,2}$ they are diagnosed with higher grade/stage prostate carcinomas, and they are less likely to receive definitive therapy (radical prostatectomy or external beam radiation therapy). ${ }^{3-6}$

Schapira et al. $^{3}$ reported a significant racial difference in the utilization of specific treatment modalities for the treatment of localized prostate carcinoma. Using data from the Surveillance, Epidemiology, and End Results (SEER) registry between 1988 and 1989, they 
reported that African-American men were less likely to receive definitive therapy (radical prostatectomy or external beam radiation therapy) when diagnosed with localized prostate carcinoma. In addition, if men received definitive therapy, then they were less likely to undergo radical prostatectomy. Other investigators have reported similar findings. ${ }^{4-6}$ Those studies documented clear racial differences in the utilization of specific treatment modalities for localized/regional prostate carcinoma. During the 1990s, the use of brachytherapy increased, and there was an effort to promote prostate cancer education, earlier detection, and definitive treatment among African-American men. ${ }^{7,8}$ The influence of those factors on racial and ethnic prostate carcinoma treatment trends remains unclear.

In contrast to the clear racial differences found in treatment for prostate carcinoma between AfricanAmerican men and white men, little has been published regarding Hispanic men. However, Hispanics comprise $6 \%$ of the United States population, and they are the largest growing minority group. Although studies have described an increase in mortality from prostate carcinoma in this population, ${ }^{9-11}$ treatment trends in the Hispanic community have not been well documented. The objective of this study was to describe racial and ethnic trends from 1992 through 1999 in the utilization of specific treatment modalities (androgen-deprivation therapy [ADT]/ expectant management, radical prostatectomy, external beam radiation therapy, brachytherapy, and combination therapy) for localized/regional prostate carcinoma among white, Hispanic, and African-American men using data from the national SEER registry.

\section{MATERIALS AND METHODS Setting}

The National Cancer Institute developed and maintains the SEER Program, a population-based cancer program, to collect incidence, treatment, and cancer mortality data. ${ }^{12}$ SEER encompasses approximately $14 \%$ of the United States population from 11 defined geographic regions, including 5 states (Connecticut, Hawaii, Iowa, New Mexico, and Utah) and 6 metropolitan regions (San Francisco-Oakland, Metropolitan Detroit, Metropolitan Atlanta, San Jose-Monterey, Seattle-Puget Sound, and Los Angeles). ${ }^{13}$ SEER cancer incidence, mortality, and demographic data generally are representative of the national population. ${ }^{13,14}$ Data collected within SEER predominately are hospitalbased inpatient and outpatient data, but they also include physician office, nursing/convalescent home/ hospice, autopsy, and death certificate information. SEER explicitly reviews diagnoses from hospital dis- charge reports, pathology reports, and death certificates. Data accuracy and completeness are evaluated through a routine quality-control program..$^{13}$ Patient demographics, disease stage, first course of therapy, and survival data are available from the SEER registries. $^{12}$

\section{Identification of Patients with Prostate Carcinoma}

To evaluate racial and ethnic trends in the utilization of specific therapies for localized/regional prostate carcinoma, data were abstracted from the available SEER public-use tapes and all incident diagnoses ( $n$ $=161,289 \mathrm{men})$ of localized/regional prostate carcinoma (International Classification of Diseases, ninth revision; code 185) histologically identified as adenocarcinoma (International Classification of Diseases for Oncology, second revision; code 8140/3) were identified. SEER defines localized disease as invasive carcinoma of any size confined to the organ of origin and defines regional disease as extending beyond the limits of the organ or origin and/or involving the regional lymph nodes but excluding distant lymph nodes. ${ }^{12}$ Within SEER, the most complete staging information is used, incorporating data from pathologic (i.e., radical prostatectomy) and clinical staging. ${ }^{12}$ In addition, within SEER, individual race identity is determined from self-reported demographic information within medical records. ${ }^{12}$ Of the previously identified incident diagnoses, we excluded 5482 men (3.4\%) due to a lack of data on race and/or treatment received, and we excluded 5351 men (3.3\%) due to a lack of tumor grade information. The 3 largest racial and ethnic groups from SEER are white, Hispanic, and African American, accounting for $94.6 \%$ of the sample. For the purpose of this report, we restricted our analysis to these three groups. In this manner, we identified 142,340 men who were diagnosed with localized/regional prostate carcinoma, including 116,132 white men (81.6\%), 9121 Hispanic men (6.4\%), and 17,087 African-American men $(12.0 \%)$.

\section{Definition of Interventions}

The proportion of men whose first course of therapy was ADT/expectant management, surgery, external beam radiation therapy, brachytherapy, or some combination was determined. Within SEER, the first course of therapy is defined as all disease-directed therapy within 4 months after the initial diagnosis and all planned therapy within the first year, as indicated in the medical record. We defined radical prostatectomy as undergoing surgery with curative intent; men who underwent transurethral resection of the prostate and other noncurative procedures were not included in the radical prostatectomy group. SEER does not 
TABLE 1

Distribution of Age and Grade by Race and Ethnicity ${ }^{a}$

\begin{tabular}{lllll}
\hline Characteristic & Total sample & White & Hispanic & African-American \\
\hline No. of patients (\%) & $142,340(100.0)$ & $116,132(81.6)$ & $9121(6.4)$ & $17,087(12.0)$ \\
Age in yrs (mean \pm SD) & $68.6 \pm 8.8$ & $68.9 \pm 8.7$ & $68.1 \pm 8.6$ & $66.5 \pm 9.0$ \\
Married (\%) & 78.6 & 80.8 & 77.3 & 64.3 \\
Tumor grade (\%) & 13.1 & 13.3 & 14.9 & 11.0 \\
$\quad$ Well differentiated & 67.7 & 67.9 & 64.6 & 68.0 \\
$\quad$ Moderately differentiated & 19.2 & 18.8 & 20.5 & 21.0 \\
Poorly differentiated & & & $<0.001$ \\
\hline
\end{tabular}

SD: standard deviation.

${ }^{a}$ Chi-square analysis was used to compare distribution across races, except for age, for which an analysis of variance was used.

contain information on the dose of radiation or whether the full course of therapy was completed; therefore, we defined external beam radiation therapy as the receipt of any external beam radiation. We defined brachytherapy as the receipt of radiation implants (seeds) or radioisotopes. We defined combination therapy as the receipt of any combination of radical prostatectomy, external beam radiation therapy, or brachytherapy. The ADT/expectant management group consisted of all other individuals who were not classified otherwise in the radical prostatectomy, external beam radiation therapy, brachytherapy, or combination therapy groups. Information on the utilization of $\mathrm{ADT}$ is not available in the public-use national SEER data set; therefore, men who received only ADT were included in the ADT/expectant management group.

\section{Statistical Analysis}

The primary outcome measure in this study was the utilization of definitive therapies for localized/regional stage prostate carcinoma among men who were diagnosed between 1992 and 1999. Chi-square analyses were performed to ascertain possible differences between racial and ethnic groups in the distribution of marital status, tumor grade, and treatment received (ADT/expectant management, radical prostatectomy, external beam radiation therapy, brachytherapy, or combination therapy). An analysis of variance was used to examine age differences by race and ethnicity. Logistic regression models were used to assess whether the odds of receiving a specific treatment modality differed by racial and ethnic group and whether the differences between racial and ethnic groups changed over time, adjusting for age, marital status, tumor grade, and SEER site (geography). The change in racial and ethnic differences over time was addressed by including racial and ethnic group by diagnosis year interaction terms in the model. A back- ward model-selection procedure was used to obtain the most parsimonious models. In addition, log-linear modeling was used to generate estimates of the distribution of race and ethnicity by treatment adjusted for age group $(<50$ years, 50-59 years, $60-69$ years, 70-79 years, and $\geq 80$ years), marital status, tumor grade, and SEER site. All statistical analyses were performed using SAS software (SAS Institute, Inc., Cary, NC). The level of significance for all statistical analysis was set at $P<0.05$.

\section{RESULTS}

\section{Cohort Characteristics}

Among all 142,340 men in the cohort, $81.6 \%$ were white, $6.4 \%$ were Hispanic, and $12.0 \%$ were African American. Compared with Hispanic and white men, African-American men were diagnosed at a significantly younger age. They also were diagnosed in greater proportion with moderate and poorly differentiated tumors, and they were married in lower proportion (Table 1).

\section{Racial and Ethnic Differences in Treatment}

We determined the adjusted estimates of receiving a specific treatment modality between 1992 and 1999 stratified by race and ethnicity. In this model, estimates were adjusted for age, marital status, tumor grade, and SEER site (Table 2). Of the treatment modalities evaluated, white and Hispanic men were more likely to undergo radical prostatectomy, whereas African-American men were more likely to receive ADT/ expectant management $(P<0.001)$. Compared with white men, Hispanic and African-American men were significantly more likely to receive ADT/expectant management $(P<0.001)$. Hispanic men were more likely to undergo radical prostatectomy than both white and African-American men $(P<0.001)$. Compared with white and Hispanic men, African-American men were more likely to receive external beam 
TABLE 2

Log-Linear Adjusted Estimates of Distribution of Specific Treatment Modalities Stratified by Race and Ethnicity for the Period 1992-1999a

\begin{tabular}{lllll}
\hline & \multicolumn{3}{c}{ Adjusted \% } \\
\cline { 2 - 4 } & & & African- & \\
Therapy & Whites & Hispanics & Americans & P value \\
\hline ADT/expectant & & & & \\
$\quad$ management & 29.6 & 33.4 & 34.5 & $<0.001$ \\
Radical prostatectomy & 36.7 & 40.1 & 31.7 & \\
External beam radiation & 23.8 & 20.7 & 26.0 & \\
Brachytherapy & 3.8 & 1.5 & 2.3 & \\
Combination therapy & 6.1 & 4.3 & 5.5 & \\
\hline
\end{tabular}

ADT: androgen-deprivation therapy.

Log-linear regression models adjusted for age, marital status, tumor grade, and SEER site (geography) were used to determine the adjusted estimates of use of specific treatments across racial and ethnic groups. All pairwise comparisons between racial treatment distributions were statistically significant at $P<0.001$.

radiation therapy $(P<0.001)$. Compared with the other treatment modalities, among the 3 groups, brachytherapy was used the least; however, white men were most likely, and Hispanic men were least likely, to receive brachytherapy $(P<0.001)$. Although the proportion of our cohort that received combination therapy was relatively low, compared with Hispanic and African-American men, white men were more likely to receive combination therapy $(P<0.001)$.

\section{Changes Over Time}

To determine whether the utilization of specific treatment modalities for localized/regional prostate carcinoma changed over the study period, we examined the adjusted trends in utilization of ADT/expectant management, radical prostatectomy, external beam radiation therapy, brachytherapy, and combination therapy per 1000 men with prostate carcinoma who were diagnosed between 1992 and 1999, stratified by race and ethnicity (Fig. 1). Within the study cohort, the utilization of ADT/expectant management decreased significantly over time $(P<0.001)$. Racial and ethnic differences in the rates of use of ADT/expectant management were found; however, those differences appeared to diminish over time. In 1992, white men received $\mathrm{ADT} /$ expectant management at a rate that was statistically significantly lower compared with Hispanic and African-American men. Although the racial and ethnic differences in the utilization of ADT/ expectant management improved by 1999, white men still had significantly lower utilization rates than African-American men. However, in 1999, the utilization of $\mathrm{ADT} /$ expectant management was not statistically significantly different between white men and Hispanic men (Fig. 1A).

Throughout the study period, the rate of utilization of radical prostatectomy increased for Hispanic men, remained relatively flat for African-American men, and generally decreased for white men. Overall, Hispanic men had the highest rate of utilization of radical prostatectomy, and African-American men had the highest rate of utilization of external beam radiation therapy (Table 2). The rate utilization of external beam radiation therapy generally increased for Hispanic men and generally decreased for African-American and white men; such that, by 1999, differences in the use of external beam radiation therapy for white, Hispanic, and African-American men were not statistically significant. $(P>0.05)$ (Fig. 1C). Although the rates of use of brachytherapy and combination therapy were low, they increased for white, Hispanic, and African-American men between 1995 and 1999. It is noteworthy that white men had the highest rates and Hispanic men had the lowest rates of utilization of both brachytherapy and combination therapy ( $P$ $<0.001$ ) (Fig, 1D,E).

\section{DISCUSSION}

In the current study, we examined racial and ethnic differences in the utilization of specific treatment modalities by men diagnosed with localized/regional prostate carcinoma. We found that, compared with white men, Hispanic and African-American men were more likely to receive ADT/expectant management. Consistently, African-American men were more likely to receive ADT/expectant management and external beam radiation therapy and were less likely to undergo radical prostatectomy compared with white and Hispanic men. This is in agreement with the results of Schapira et al., ${ }^{3}$ who found that African-American men were $46 \%$ as likely as white men to undergo radical prostatectomy or receive external beam radiation therapy. Those authors also reported that, among men who either underwent radical prostatectomy or received external beam radiation therapy, AfricanAmerican men were $64 \%$ less likely than white men to undergo radical prostatectomy. Harlan et al., ${ }^{15}$ in a later study, reported that African-American men age $\geq 60$ years underwent aggressive treatment (radical prostatectomy or external beam radiation therapy) less often than white or Hispanic men. There are a growing number of studies that find racial differences in the receipt of major therapeutic procedures for a broad range of conditions, even after adjustment for insurance status, economic status, and severity of disease. $^{16-18}$

Given the lack of scientific data of the best specific 
ADT/Expectant Management

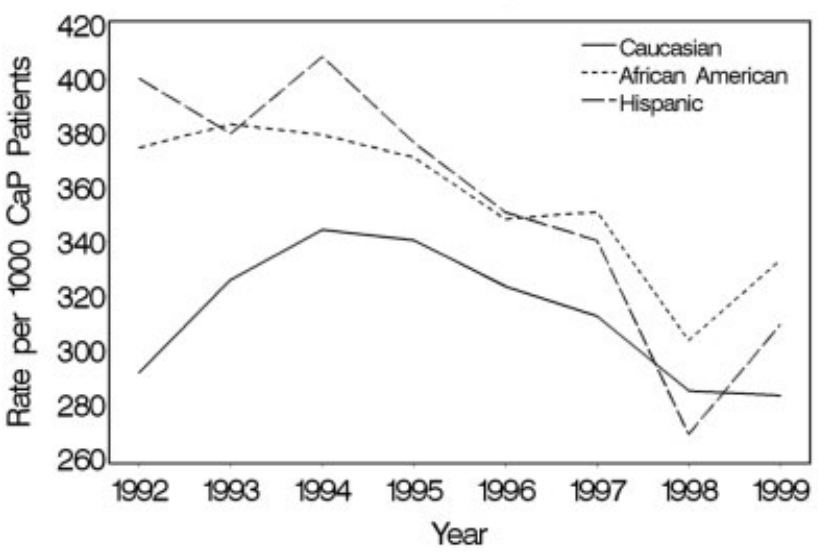

External Beam Radiation

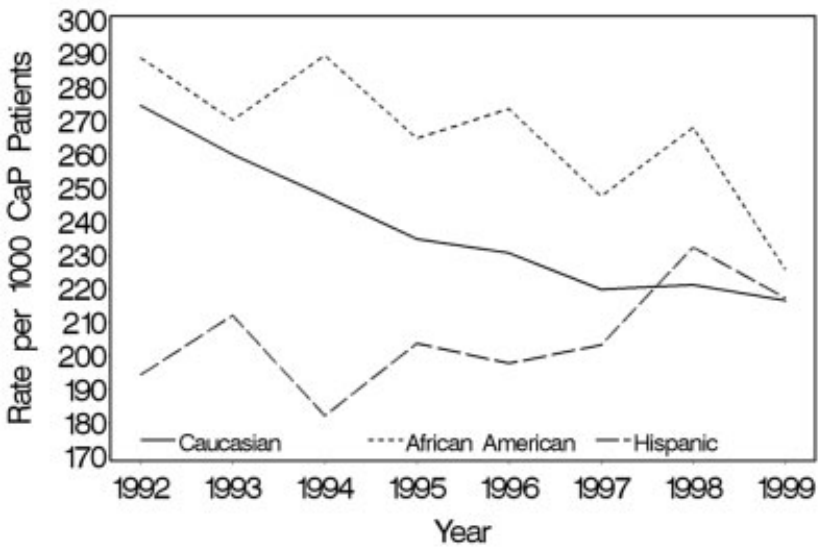

Radical Prostatectomy

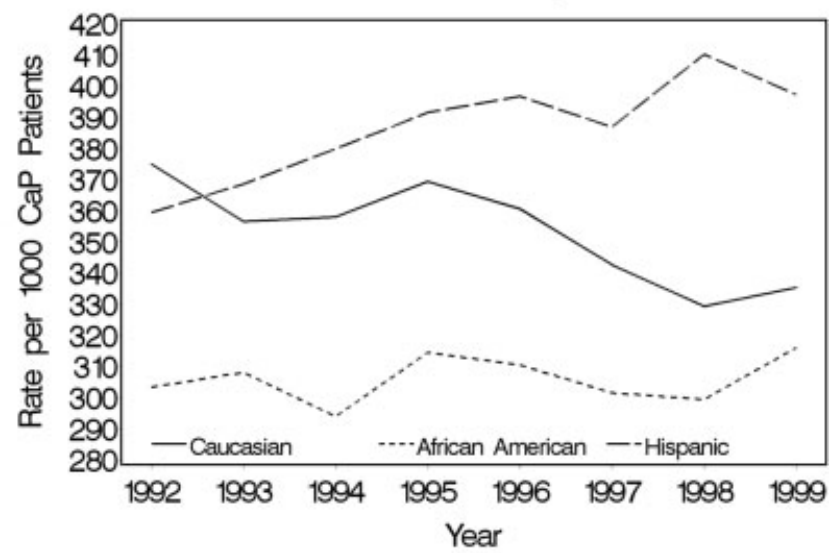

Brachytherapy

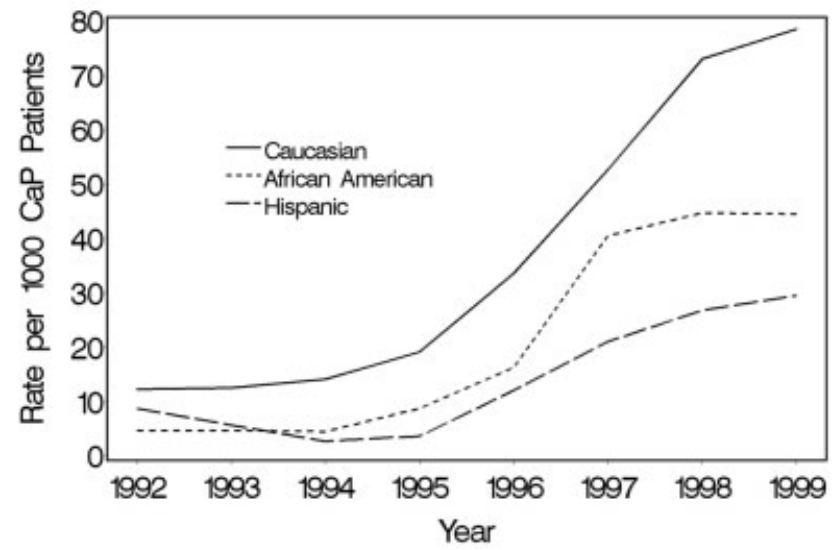

\section{Combination Therapy}

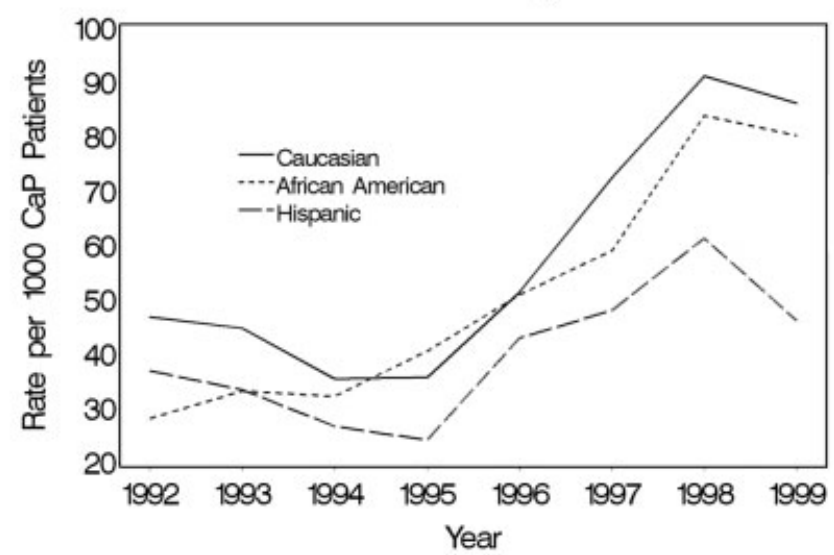

FIGURE 1. Trends of utilization of androgen-deprivation therapy (ADT)/expectant management (A), radical prostatectomy (B), external beam radiation therapy (C), brachytherapy (D), and combination therapy (E) stratified by race and ethnicity for white, Hispanic, and African-American patients with prostate carcinoma (CaP) for the period 1992-1999. Logistic regression analyses were used to determine whether the odds of receiving ADT/expectant management, radical prostatectomy, external beam radiation therapy, brachytherapy, or combination therapy differed by racial and ethnic group and whether the differences between racial and ethnic groups changed over time, adjusted for age, marital status, tumor grade, and SEER site. The changes in racial and ethnic differences over time were determined by including race/ethnic group by year of diagnosis interaction terms in the model. 
treatment modality for localized/regional prostate carcinoma, treatment decision-making can be difficult both for patients and for providers. Patient-level variables (e.g., socioeconomic status, preferences toward treatment outcomes, and trust in the healthcare system) and provider-level variables (e.g., clinician biases toward specific treatment recommendations and the lack of clinician agreement on what information is essential in making a treatment decision) are possible confounders in the treatment decision-making process for men with localized/regional prostate carcinoma. ${ }^{19-30}$ Therefore, it is important to improve our understanding of how patient-level and provider-level variables may confound patient racial and ethnic differences in the treatment received for localized/regional prostate carcinoma.

\section{Patient-Level Variables}

The outcomes reported here, as noted above, should be interpreted with respect to the influence of patients' socioeconomic status, preferences toward treatment outcomes, and trust in the healthcare system. Because race/ethnicity and socioeconomic position are intertwined closely, it is difficult to determine the extent to which the reported racial and ethnic healthcare differences are due to differences in socioeconomic status. ${ }^{31}$ Morris et al. ${ }^{19}$ evaluated racial and ethnic differences in the use of radical prostatectomy, adjusting for socioeconomic status. They reported that, compared with white men, African-American men were less likely, and Hispanic men were equally as likely, to undergo radical prostatectomy. Those authors concluded that, although differences in socioeconomic status influenced treatment received (with men of lower income levels less likely to undergo radical prostatectomy), they did not explain entirely the differences found between African-American men and white men.

Racial and ethnic differences in the preference toward treatment outcomes are not well understood. Steginga et al. $^{32}$ reported that prostate carcinoma treatment decisions were influenced primarily by nonsystematic decision processes, such as deferral to physicians' recommendation, the positive and negative experiences of others, and preexisting belief that surgery is a better treatment for prostate carcinoma. When patients did utilize a systematic approach, however, they most commonly reported incontinence $(55 \%)$ and impotence $(51 \%)$ from surgery and bowel problems $(30 \%)$ and skin burning $(21 \%)$ from radiation therapy as factors that influenced their treatment decision. O'Rouke and Germino, ${ }^{33}$ in a focus group study, reported that beliefs about cancer and possible cures were major factors in the treatment decisions among men and their spouses who had been treated previously for localized prostate carcinoma. Although those studies have contributed to our understanding of the patient-level variables that influence treatment decisions, they did not include racially and ethnically diverse populations. Therefore, it is unknown whether the findings to date are the result of racial and ethnic differences in risk aversion to treatment side effects, beliefs about cancer and cure, or historic experiences in the healthcare system that influence treatment preferences.

Racial and ethnic differences regarding mistrust of the healthcare system are well documented. ${ }^{25,34}$ Corbie-Smith et al. ${ }^{25}$ reported that African Americans were more likely than whites not to trust that their physicians would explain research participation fully. Those authors also reported that African Americans were more likely to believe that their physician sometimes exposed them to unnecessary risks and used them as "guinea pigs" without their consent. Very little is known about racial and ethnic differences in mistrust of physicians and prostate carcinoma treatment. Based on the general literature, it is reasonable to speculate that minority patients' mistrust may result in greater refusal of more invasive procedures, such as radical prostatectomy. It should be noted, however, that, irrespective of the higher level of reported mistrust of the medical community by racial and ethnic minorities, most of these patients are satisfied with and have confidence in their healthcare providers. ${ }^{34,35}$ Furthermore, mistrust or perceived discrimination alone reportedly is unlikely to cause ethnic minority patients to reject lifesaving or highly recommended procedures that promise to improve health. ${ }^{34}$ Further research is needed in this area to determine the possible influence of patient mistrust in the treatment of localized/regional prostate carcinoma, particularly as it relates to Hispanic and African-American men.

\section{Provider-Level Variables}

Due to the lack of a randomized clinical trial showing a clear survival advantage for any given treatment, objectively advising patients in the optimal treatment for localized/regional prostate carcinoma is difficult. The lack of evidence for an optimal treatment modality may contribute to the findings of Fowler et al., ${ }^{29}$ who reported that radiation oncologists and urologists tended to recommend the therapy that they themselves deliver. In a head-to-head comparison of surgery versus external beam radiation therapy, urologists overwhelmingly reported that surgery was better. ${ }^{29}$ Urologists and radiation oncologists not only disagree on which prostate treatment is best, they also disagree on what information should be discussed 
with the patients during consultation for prostate carcinoma treatment. ${ }^{28}$ These reported disagreements among physicians imply that clinicians may be imposing their own personal values when making treatment recommendations and when deciding what information should be discussed with the patient. It is unknown whether these clinician treatment biases are influenced by patients' race and ethnicity or the possible affect on clinical practice. Therefore, it is difficult to determine the extent, if any, to which these reported clinician biases have contributed to racial and ethnic differences in prostate carcinoma treatment.

Another factor that may influence racial and ethnic differences in the utilization of ADT/expected management, radical prostatectomy, and external beam radiation therapy is the increased utilization of brachytherapy and combination therapy during the study period. The overall utilization of brachytherapy was low: $3.8 \%$ among white men, $1.5 \%$ among Hispanic men, and $2.3 \%$ among African-American men. The rates of utilization, however, increased significantly from 1992 to 1999 for all 3 groups. These results are consistent with the findings of Mettlin et al., ${ }^{7}$ who reported an increase in the utilization of brachytherapy from 1992 to 1996. The use of ADT/expectant management decreased from 1994 to 1999 for the 3 groups. During the same period, white men had decreased utilization of radical prostatectomy and external beam radiation therapy and had increased utilization of combination therapy and brachytherapy. It appears that, for white men, brachytherapy may have influenced significantly the decline in the use of ADT/ expectant management, radical prostatectomy, and external beam radiation therapy from 1994 through 1999.

Although the selection of brachytherapy showed an overall increase during the study period, it showed the greatest increase among white men. It is claimed that the efficacy of brachytherapy is equivalent to that of external beam radiation therapy and radical prostatectomy in men who have a low risk of biochemical failure (prostate-specific antigen $\leq 10 \mathrm{ng} / \mathrm{mL}$ and Gleason score $\leq 6) .{ }^{36}$ However, African-American and Hispanic men within the current study population had a higher proportion of poor-grade tumors; possibly making brachytherapy a less ideal treatment modality for many of them. Thus, our findings of lower utilization of brachytherapy by African-American and Hispanic men may be the result of clinical parameterappropriate utilization of brachytherapy.

In interpreting the results of the current study, several limitations should be considered. First, within SEER, treatment is defined as received or planned to receive within the first year of diagnosis. Thus, delays in treatment due to neoadjuvant ADT beyond this time may be misclassified as ADT/expectant management. This is unlikely, however, because the typical use of neoadjuvant ADT is only 3-6 months. Therefore, subsequent intent to receive a specific definitive therapy should have been identified by SEER as planned in the first year after diagnosis. Second, differential rates of the utilization of radical prostatectomy among African-American men may be secondary to higher rates of positive lymph nodes during radical prostatectomy and subsequent abortion of procedure. Such patients would be captured only as ADT/expectant management, resulting in potential bias. To examine this further, we performed a secondary analysis on respondents from 1998 and 1999, for which there were available SEER data on pelvic lymph dissection, to evaluate for any racial and ethnic disparities among men who underwent pelvic lymph node dissection without radical prostatectomy. In that analysis, we did not observe significant differences among racial or ethnic groups $(P>0.05)$; therefore, it is unlikely that our findings of lower rates of use of radical prostatectomy by African-American men were influenced by higher rates of positive lymph nodes during radical prostatectomy and subsequent abortion of procedure in African-American men.

In conclusion, during the study period, the overall the utilization of ADT/expectant management decreased for all three groups; Hispanic and AfricanAmerican men were more likely to have received ADT/ expectant management compared with white men; however, this racial and ethnic difference decreased over time. Hispanic men were most likely to undergo radical prostatectomy, and African-American men were most likely to receive external beam radiation. However, the utilization of external beam radiation among African-American men decreased over the study period. The utilization of brachytherapy and combination therapy increased for all three groups, with the greatest increase observed among white men. To ascertain the implications of our findings for racial and ethnic differences in the treatment of localized/ regional prostate carcinoma, further research will be required to isolate possible racial and ethnic differences, like those observed in the current study, in the patient-level and provider-level variables that may influence prostate carcinoma treatment decisions.

\section{REFERENCES}

1. Jemal A, Murray T, Samuals A, Ghafoor A, Ward E, Thun M. Cancer statistics, 2003. CA Cancer J Clin. 2003;53:5-26.

2. Landis SH, Murray T, Bolden S, Wingo PA. Cancer statistics, 1999. CA Cancer J Clin. 1999;49:8-31. 
3. Schapira MM, McAuliffe TL, Nattinger AB. Treatment of localized prostate cancer in African-American compared with Caucasian men. Less use of aggressive therapy for comparable disease. Medical Care. 1995;33:1079-1088.

4. Mettlin CJ, Murphy GP, McGinnis LS, Menck HR. The National Cancer Data Base report on prostate cancer. American College of Surgeons Commission on Cancer and the American Cancer Society. Cancer. 1995;76:1104-1112.

5. Mettlin CJ, Murphy GP, Cunningham MP, Menck HR. The National Cancer Data Base report on race, age, and region variations in prostate cancer treatment. Cancer. 1997;80: 1261-1266.

6. Harlan L, Brawley O, Pommerenke F, Wali P, Kramer B. Geographic, age, and racial variation in the treatment of local/regional carcinoma of the prostate. J Clin Oncol. 1995; 13:93-100.

7. Mettlin CJ, Murphy GP, McDonald CJ, Menck HR. The National Cancer Data Base Report on increased use of brachytherapy for the treatment of patients with prostate carcinoma in the U.S. Cancer. 1999;86:1877-1882.

8. Powell I, Gelfand D, Parzuchowski M, Heilbrun L, Franklin A. A successful recruitment process of African American men for early detection of prostate cancer. Cancer. 1995; 75(Suppl):1880-1884.

9. Gilliland FD, Becker TM, Key CR, Samet JM. Contrasting trends of prostate cancer incidence and mortality in New Mexico's Hispanics, non-Hispanic whites, American Indians, and blacks. Cancer. 1994;73:2192-2199.

10. Canto MT, Chu KC. Annual cancer incidence rates for Hispanics in the United States: Surveillance, Epidemiology, and End Results, 1992-1996. Cancer. 2000;88:2642-2652.

11. Gilliland FD, Welsh DJ, Hoffman RM, Key CR. Rapid rise and subsequent decline in prostate cancer incidence rates for New Mexico, 1989-1993. Cancer Epidemiol Biomarkers Prev. 1995;4:797-800.

12. SEER. SEER Program code manual, 3rd ed. Bethesda: National Cancer Institute, 1998.

13. Hankey BF, Ries LA, Edwards BK. The Surveillance, Epidemiology, and End Results Program: a national resource. Cancer Epidemiol Biomarkers Prev. 1999;8:1117-1121.

14. Frey C, McMillen M, Cowen C, Horn J, Kessler L. Representativeness of the Surveillance, Epidemiology, and End Result Program data: recent trends in cancer mortality rates. J Natl Cancer Inst. 1992;872-877.

15. Harlan LC, Potosky A, Gilliland FD, et al. Factors associated with initial therapy for clinically localized prostate cancer: Prostate Cancer Outcomes Study. J Natl Cancer Inst. 2001; 93:1864-1871.

16. McBean AM, Gornick M. Differences by race in the rates of procedures performed in hospitals for Medicare beneficiaries. Health Care Financing Rev. 1994;15:77-90.

17. Hannan E, van Ryn M, Burke J, et al. Access to coronary artery bypass surgery by race/ethnic and gender among patients who appropriate for surgery. Medical Care. 1999;37: 68-77.

18. Harris DR, Andrews R, Elixhauser A. Racial and gender differences in use of procedures for black and white hospitalized adults. Ethn Dis. 1997;7:9-105.
19. Morris CR, Snipes KP, Schlag R, Wright WE. Sociodemographic factors associated with prostatectomy utilization and concordance with the physician data query for prostate cancer (United States). Cancer Causes Control. 1999;10:503511.

20. Klabunde CN, Potosky AL, Harlan LC, Kramer BS. Trends and black/white differences in treatment for nonmetastatic prostate cancer. Medical Care. 1998;36:1337-1348.

21. Greenwald HP, Henke CJ. HMO membership, treatment, and mortality risk among prostatic cancer patients. Am J Public Health. 1992;82:1099-1104.

22. Potosky AL, Harlan LC, Stanford JL, et al. Prostate cancer practice patterns and quality of life: the Prostate Cancer Outcomes Study. J Natl Cancer Inst. 1999;91:1719-1724.

23. Mazur DJ, Merz JF. Older patients' willingness to trade off urologic adverse outcomes for a better chance at five-year survival in the clinical setting of prostate cancer. J Am Geriatr Soc. 1995;43:979-984.

24. Lubeck DP, Grossfeld GD, Carroll PR. A review of measurement of patient preferences for treatment outcomes after prostate cancer. Urology. 2002;60:72-77; discussion, 77-78.

25. Corbie-Smith G, Thomas SB, St Georg DM. Distrust, race, and research. Arch Intern Med. 2002;162:2458-2463.

26. Leak B, Wei JT, Gabel M, et al. Relevant patient and tumor considerations for early prostate cancer treatment. Semin Urol Oncol. 2002;20:39-44.

27. O'Rourke ME. Decision making and prostate cancer treatment selection: a review. [Review; 50 references.] Semin Oncol Nurs. 2001;17:108-117.

28. Feldman-Stewart D, Brundage MD, Hayter C, et al. What prostate cancer patients should know: variation in professionals' opinions. Radiother Oncol. 1998;49:111-123.

29. Fowler FJ Jr., McNaughton Collins M, Albertsen PC, Zietman A, Elliott DB, Barry MJ. Comparison of recommendations by urologists and radiation oncologists for treatment of clinically localized prostate cancer. JAMA. 2000;283:3217-3222.

30. Deber RB, Kraetschmer N, Irvine J. What role do patients wish to play in treatment decision making? Arch Intern Med. 1996;156:1414-1420.

31. Fiscella K, Franks P, Gold MR, Clancy CM. Inequality in quality: addressing socioeconomic, racial, and ethnic disparities in health care. JAMA. 2000;283:2579-2584.

32. Steginga SK, Occhipinti S, Gardiner RA, Yaxley J, Heathcote P. Making decisions about treatment for localized prostate cancer. BJU Int. 2002;89:255-260.

33. O'Rourke ME, Germino BB. Prostate cancer treatment decisions: a focus group exploration. Oncol Nurs Forum. 1998; 25:97-104.

34. Smedley B, Stith A, Nelson A. Unequal treatment: confronting racial and ethnic disparities in health care. Washington, DC: The National Academies Press, 2002.

35. Shi L. Experience of primary care by racial and ethnic groups in the United States. Medical Care. 1999;37:10681077.

36. D'Amico AV, Whittington R, Malkowicz SB, et al. Biochemical outcome after radical prostatectomy, external beam radiation therapy, or interstitial radiation therapy for clinically localized prostate cancer. JAMA. 1998;280:969-974. 